INPLASY

PROTOCOL

To cite: Li et al. The profiles of proinflammatory cytokines and T cell in patients with Tourette syndrome: A meta-analysis. Inplasy protocol 2021110079. doi:

10.37766/inplasy2021.11.0079

Received: 22 November 2021

Published: 22 November 2021

Corresponding author:

Yonghua Cui

cuiyonghua@bch.com.cn

Author Affiliation:

Department of Psychiatry, Beijing

Children's Hospital, Capital

Medical University, National

Centre for Children's Health,

Beijing.

Support: No financial support.

Review Stage at time of this submission: The review has not yet started.

Conflicts of interest:

None declared.

\section{The profiles of proinflammatory cytokines and $T$ cell in patients with Tourette syndrome: A meta-analysis}

Li, Y1; Wang, X2; Gui, J3; Cui, Y4.

Review question / Objective: The aim of this meta-analysis is to investigate the profiles of $T$ cell and proinflammatory cytokines by meta-analysis in patients with TS. Based on these results, we might identify confirmed evidence of immune dysregulation for TS.

Eligibility criteria: The Inclusion Criteria were as follows:(1) English or Chinese language studies from peer-reviewed journals. (2) The included patients were diagnosed as the Tourette syndrome related disorders. (3) The $T$ cell and cytokines was assessed by serum orplasma of peripheral blood.Exclusion criteria were as follows:(1) Cases report or review or meta-analysis.(2) The sample size was less than 5. (3) Studies involved Rat or mouse rather than human beings. (4) Studies were based on same sample size.

INPLASY registration number: This protocol was registered with the International Platform of Registered Systematic Review and Meta-Analysis Protocols (INPLASY) on 21 November 2021 and was last updated on 21 November 2021 (registration number INPLASY2021110079).

\section{INTRODUCTION}

Review question / Objective: The aim of this meta-analysis is to investigate the profiles of $\mathbf{T}$ cell and proinflammatory cytokines by meta-analysis in patients with TS. Based on these results, we might identify confirmed evidence of immune dysregulation for TS.
Condition being studied: In our recent report for the prevalence of tic disorders in China, it was $\mathbf{2 . 5 \%}$. Tourette syndrome (TS) is one of the three types of tic disorders which showed more persistence of tic symptoms. However, the aetiology of TS is still not well-established, and the pathophysiology of TS is still needed further exploration. Numerous studies tried 
to investigate the pathophysiology of TS from the behavioural to imaging level, and from the genetic level to the immunological level. It should be noted that immune dysfunction was one of the important dimensions to investigate the TS. Tic disorder is a neurodevelopmental disorder characterized by motor and phonic tics symptoms. Tourette syndrome (TS) is one of the three types of tic disorders which showed more persistence of tic symptoms. The immune dysfunction for the TS was still unclear due to the limited evidence, especially for the paediatric patients. Based on recent reports, it suggested that the infection factors might be one of the triggers for the fluctuation of tic symptoms. It also provides us with an important perspective to investigate the immune dysfunctions of TS. Furthermore, no matter the innate immunity or the adaptive immunity, the increased proinflammatory cytokines can be identified in many patients with TS. It should be noted that previous studies in patients with TS have observed an increased level of proinflammatory cytokines, including TNF$a$ and ILs, such as IL-2, IL-6, IL-8, IL-12. However, most of these studies were limited by a small sample size and more confirmed evidence was needed.

\section{METHODS}

Search strategy: A total of five databases including PubMed, Web of Science, PsycINFO, Google Scholar and the China National Knowledge Infrastructure were searched.

Participant or population: Pediatric patients with Tourette syndrome.

Intervention: This study was a crosssectional meta-analysis without intervention.

Comparator: This study was a crosssectional meta-analysis without comparator.

Study designs to be included: A metaanalysis will be carried out based on the data of published articles to identify the characteristics of $T$ cells and proinflammatory cytokines in pediatric patients with Tourette syndrome.

Eligibility criteria: The Inclusion Criteria were as follows:(1) English or Chinese language studies from peer-reviewed journals. (2) The included patients were diagnosed as the Tourette syndrome related disorders. (3) The $T$ cell and cytokines was assessed by serum orplasma of peripheral blood.Exclusion criteria were as follows:(1) Cases report or review or meta-analysis.(2) The sample size was less than 5. (3) Studies involved Rat or mouse rather than human beings.(4) Studies were based on same sample size.

Information sources: A systematic search was performed in PubMed, Elsevier, and China National Knowledge Infrastructure (CNKI).

Main outcome(s): Based on the inclusion and exclusion criteria, a total of identified 25 studies were included in this metaanalysis (For more details see Figure 1). There were 14 studies report the CD3+ level, CD4+ level, CD8+ level and 12 studies for CD4+/CD8+. There were 4 studies for IL-2, 4 studies for IL-4, 8 studies for IL-6, 4 studies for IL-8, 5 studies for IL-12, 4 studies for INF-, 9 studies for TNF-a. We summarized the expression of several proinflammatory cytokines and $T$ cell in TS patient and healthy controls. All included studies measured cytokines in serum from drug-free TS participants at the time of sample collection. The expression of seven cytokines (TNF-a, IL-6, IL-12, IL-8, IL-2, IL-4 and IFN- $r$ ) and three types of $\mathrm{T}$ cell (CD3+, CD4 + , CD8 +, CD4 + / CD8+) were summarized. Furthermore, we list the authors, publication years, countries, sample sizes (Patients/control), mean ages(years), types of proinflammatory cytokines and T cell, YGTSS scores (mean sd), technique for each included study.

Quality assessment / Risk of bias analysis: The JBI scales for each included study was included in the Table 2. All the studies were at least have five "Yes". We publication bias were assessed by the Egger test. There 
was no publication bias was identified for all meta-analysis ( $p>0.05$ ) (For more details see Supplemental Material sTable 1 and sFigure 1). We used sensitivity analysis to explore the heterogeneity of the pooled SMD. The results showed that there are no studies 12 changes more than $5 \%$ (Supplemental Materials Figure 2).

Strategy of data synthesis: The 12 and forest plots were used to identify the heterogeneity of the meta-analysis. If 12 was greater than $50 \%$, we used a randomeffects model. SMD was used for the effect size of the comparisons in each included study. Egger test is also calculated to judge whether there is publication bias. The sensitivity analysis were also performed (omits one study at a time and tracks the change in 12 to identify the contribution of each study to the heterogeneity). We then performed subgroup analysis and metaregression analyses to explore heterogeneities in the effect size for this meta-analysis. We considered a p-value < 0.05 to be statistically significant, and all the analyses were performed in $\mathbf{R}$ (version 3.5.3) using the "meta" or "metafor" packages.

Subgroup analysis: The effects of age were analyzed by subgroup analysis.

Sensitivity analysis: The sensitivity analysis was also performed (omits one study at a time and tracks the change in 12 to identify the contribution of each study to the heterogeneity). We used sensitivity analysis to explore the heterogeneity of the pooled SMD. The results showed that there are no studies 12 changes more than $5 \%$ in the meta-analysis of IL-2, IL-6, IL-8, IL-12, CD4+, CD8+.

Language: Chinese and English.

Country(ies) involved: China.

Keywords: Tourette syndrome, proinflammatory cytokines, T cell, Immunological Dysfunction, metaanalysis
Contributions of each author:

Author 1 - Ying Li.

Email: liying@bch.com.cn

Author 2 - Xiaolin Wang.

Email: wangxl19891012@163.com

Author 3 - Jingang Gui.

Email: guijingang@bch.com.cn

Author 4 - Yonghua Cui.

Email: cuiyonghua@bch.com.cn 